\title{
Selective attending changes during concept learning, extinction, and superstition ${ }^{1}$
}

\author{
SVEN RYDBERG and PETER $W$. \\ ARNBERG ${ }^{2}$ \&bo Academy, \&bo, Finland, \\ and University of Stockholm, Sweden
}

Before 48 human adults began to classify stimuli correctly by a rule we withheld, they suddenly-in their trial of last error-spent significantly more time (per cent) observing relevant stimuli. Then, relevant observing increased during overlearning, decreased drastically during extinction, and again increased under a superstition condition. Ss gave more absolute observing time to closer stimuli.

Research on concept and discrimination learning has given a good deal of indirect evidence that selective attention decisively affects such measures as number of errors and trials required to reach a learning criterion (Mackintosh, 1965; Kendler \& Kendler, 1966; Trabasso \& Bower, 1968; Lovejoy, 1968). Rydberg, Kashdan, \& Trabasso (1966) described a more direct method for the study of attention. The S tactually explores separated stimulus objects and E records electrically if, when, and for how long $S$ touches each object (see also Fig. 1). Rydberg (1969) showed that already before Ss began to classify stimuli correctly, their observing behavior changed: mean per cent relevant observing increased markedly but gradually from the chance level in the last six precriterion trials (backward curve).

Rydberg (1969) had required his Ss to find out that one of three tactile dimensions was relevant and which response button to press for each stimulus intensity within it. The rule for stimulus-response pairing was not maximally simple. Here we wanted to use such a rule so as to minimize errors after the $S$ had already begun to turn more to relevant cues. Then the last error trial would be a better point of reference for any backward curves for observing. They would be more directly interpretable in terms of selective attention. We hypothesized that then the increase in relevant observing would be much more abrupt and perhaps occur entirely within the last error trial. We also wanted to see whether subsequent overlearning, extinction, and superstition conditions would affect observing.

The Ss were to try to find out which one of four tactile stimulus dimensions was relevant and to press one of three response buttons for the corresponding intensity levels within that dimension (see Fig. 1). Each S went through: (1) Pretraining A,
(2) Pretraining B, (3) learning, (4) extinction, and (5) superstition conditions. When $S$ pressed, a lamp at once indicated the correct response, except in (4-5).

\section{SUBJECTS}

Forty-eight students (24 males and 24 females) from an introductory educational psychology course at the University of Stockholm served as Ss.

\section{APPARATUS}

(See Fig. 1.) The experimental stimuli were contiguous to the upper surface of square blocks, $20 \times 37 \times 37 \mathrm{~mm}$, fastened in four holes $10 \mathrm{~mm}$ from each other. Touch and classification responses and their durations were recorded. Rydberg et al (1966) gives more apparatus details (changed circuitry has eliminated a temperature stability requirement).

\section{STIMULI AND POSITIONS}

Each object above represented one of three possible values in one of six stimulus dimensions: angle (A), circle size (C), depth (D), height (H), length (L), and roughness (R). ${ }^{3}$ We call the stimulus positions $\mathrm{a}, \mathrm{b}, \mathrm{c}$, and d (see Fig. 1).

\section{PROCEDURE AND DESIGN}

Throughout, $\mathrm{S}$ was free to feel one or more of four stimulus objects. In (1-2), the positions of the stimulus dimensions varied randomly from trial to trial, thereafter they were fixed. Thus, in the three main experiments, $S$ got to know which dimension-but not which value-he would find in each position.

Instructions and pretraining taught $S$ : (a) about the character of the stimuli;(b) to discriminate; (c) that only one dimension was relevant; (d) that in the relevant dimension the least intensive stimulus was the cue for the leftmost response button No. 1, medium for No. 2, and high for No. 3; and (e) to stop feeling the stimuli before responding.

\section{(1) Pretraining A}

The $\mathrm{E}$ acquainted $\mathrm{S}$ with the objects and all four positions in three trials, asked $S$ to feel $\mathrm{H}$ (relevant), and showed the correct button to press in three trials, but did not help in the next three. In the other three positions, stimuli from the other five dimensions varied in a fixed-random pattern.

\section{(2) Pretraining $B$}

The E gave only A, D, C, and L stimuli. A, $D$, and $C$, respectively, were relevant for one-third of the Ss. In the first three trials, E showed the correct button, then $S$ carried on unaided until five correct trials in a row.

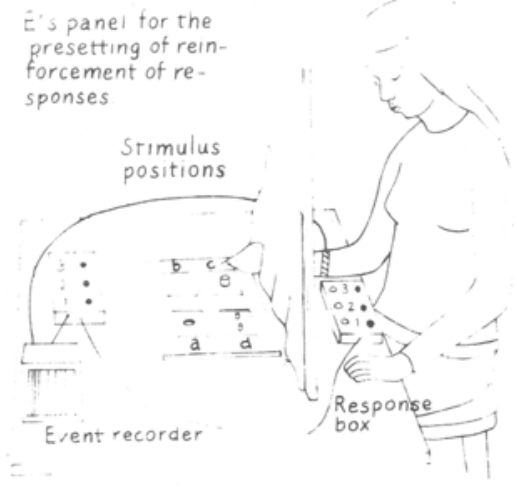

Fig. 1. Schematic apparatus description. In particular, stimuli are enlarged.

\section{(3) Learning}

From this point on, $S$ was unaided. Each $S$ 's relevant dimension-either $A, D$, or C-from (2) was replaced by R (irrelevant). One of the two other dimensions was made relevant for one-half of the Ss, the other for the rest. This made six groups of eight Ss. (Within the various groups, sex was counterbalanced.) $L$ remained and was irrelevant for all Ss. The position of each dimension was fixed for each $S$ but varied, within each group, from $S$ to $S$. This made 24 subgroups of two Ss. One $S$ in each was run to a criterion of 5 , one to 15 correct trials in a row.

\section{(4) Extinction}

As above, but $\mathrm{E}$ showed $\mathrm{S}$ that all lamps would light-up in case of a correct response. E surreptitiously cut the lamp circuits and gave $S 10$ trials without reinforcement. Touch dimensions were those from (3), except that $R$ was replaced by each $S$ 's relevant dimension $(A, D$, or $C)$ from (2). The other dimension positions we re changed diagonally from those in (3).

\section{(5) Superstition}

The E surreptitiously connected all lamp circuits and gave five trials, thus signaling "correct" regardless of the S's response; no other change was present. Analysis

Variance analyses of touching times were made for: Learning Trials 1-5, Extinction Trial 1, Extinction Trial 11 (Superstition Trial 1), and Superstition Trial 5. We also computed per cent relevant touching time, including backward curve values (Fig. 2a). RESULTS AND DISCUSSION

Throughout, no $S$ failed. Three Ss out of 48 reached criterion without any error. The slowest $S$ had 25 precriterion trials. The median was 5.5 trials.

Per Cent Relevant Touching Time

The sudden increase in Fig. 2a to the trial of last error is significant ("paired" $t=5.2$, $\mathrm{df}=42, \mathrm{p}<.001$ ). Under extinction, both overlearning and learning groups fell back to chance level observing; these groups did not 


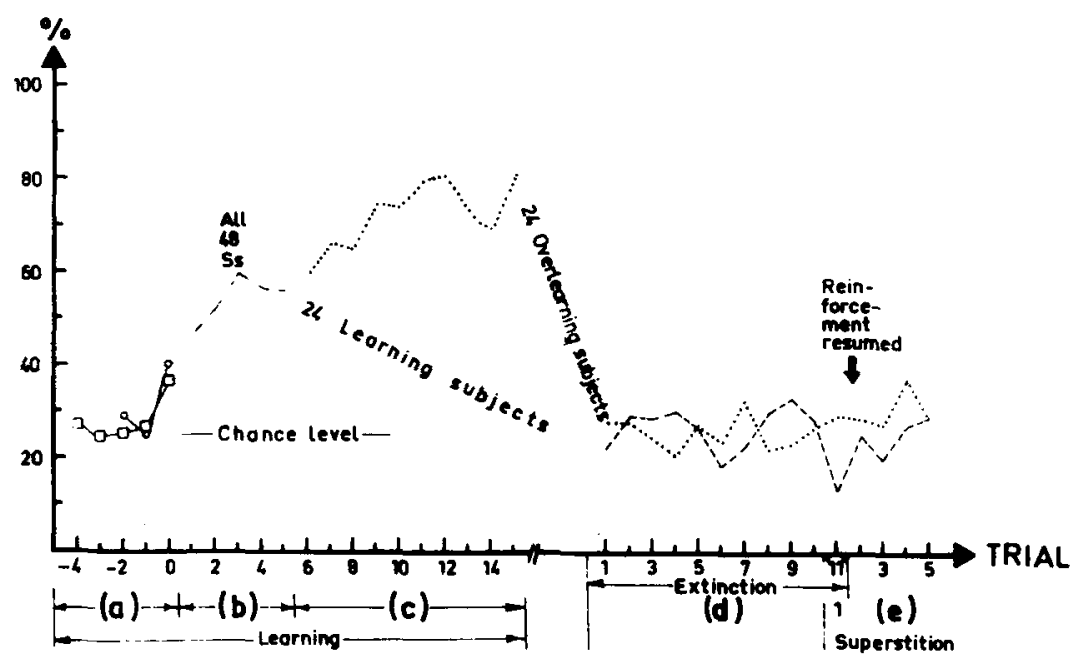

differ significantly. When any response was "correct" (superstition condition, Fig. 2e), Ss tended to revert to touching previously relevant stimuli. The mean for the Superstition Trials 2 through 5 was significantly higher than the value for "Extinction Trial 11" ("paired" $t=2.3$, $\mathrm{df}=47, p<.02$ ); group differences were not significant.

\section{Absolute Touching Times}

There were four differences at the .01 significance level (six more at .05; no significant interaction). In Learning Trial 5 , the position of the relevant dimension was a significant factor $\left(F=4.7, F_{.01}=3.9\right)$ with the longest time for Position c. In the same trial, if we disregard which position was relevant for the $S$, position by itself was a significant factor $\left(F=4.2, F_{.01}=3.9\right)$ with the longest time on Position d. In Extinction Trial 11 (i.e., Superstition Trial 1, but Ss touched before reinforcement; $F=9.0$, $F_{.01}=4.0$ ), and in Superstition Trial 5 $\left(F=4.4, F_{.01}=4.0\right)$, relevant position was again a significant factor, but only after overlearning, with the longest time for Position c.

\section{DISCUSSION}

Rydberg (1969) found, by trend analysis, significant linearly increasing trends of per cent relevant observing over the last six precriterion trials. (This also held for the
Fig. 2. Mean per cent relevant observing. (a) Precriterion trials. Backward curves for 28 Ss with more than four trials (squares) and $12 \mathrm{Ss}$ with three or four trials (circles) to criterion. (b) Criterion period. (c) Overlearning for 24 Ss. (d) Extinction. (e) Superstition trials.

time. They are easier to find and Ss touch them when passing to Positions a and $b$. Such position effects can be counteracted by tilting the stimulus board appropriately and/or through the experimental design.

In a forthcoming study, 4 the amounts of both overlearning and extinction are varied before a superstition experiment.

\section{REFERENCES \\ KENDLER, H. H., \& KENDLER, T. S. Selective}

whole period of six precriterion and 10 criterion trials.) In the present study, however, the entire precriterion increase from the chance level takes place in the trial of last error, regardless of the number of previous trials. Together, the two studies should show how changes in observing behavior precede perfect performance. When it is possible to make errors though one has already directed one's attention to a relevant stimulus dimension, the precriterion increase in relevant observing can be gradual, as in Rydberg (1969). When finding the relevant dimension is the only complication, it should be possible to show when the whole increase comes in the trial of last error, as it does here. In the terms of an old debate, it could be said (1) that the previous study gave support to continuity theory because of an artifact, and (2) that the present data give support for discontinuity theory. Let us also note that in both studies, relevant observing leveled off around $80 \%$ during overlearning though the chance level was $67 \%$ in Rydberg's experiment (1969) and only $25 \%$ here. That is, Rydberg had two relevant dimensions (redundant) out of three, and we had one out of four, but relative relevant observing remained about the same.

The Ss tended to touch the nearest positions ( $c$ and d) for a longer absolute attention versus mediation. Psychological Bulletin, 1966, 66, 282-288.

LOVEJOY, E. Attention in discrimination learning. San Francisco: Holden-Day, 1968.

MACKINTOSH, N. J. Selective attention in animal discrimination learning. Psychological Bulletin, $1965,64,124-150$.

RYDBERG, S. Concept leaming: Measured selective attending changes. Psychonomic Science, 1969, 16, 293-295.

RYDBERG, S., KASHDAN, R., \& TRABASSO, T. Recording of tactile observing responses for the study of selective attention. Psychonomic Science, 1966, 6, 197-198.

TRABASSO, T., \& BOWER, G. H. Attention in leaning: Theory and research. New York: Wiley, 1968.

1. Supported by Swedish Social Science Research Council grants.

2.P. Arnberg ran the Ss. We thank Dr. T. Trabasso, UCLA, for stimulus blocks with scaling data and $E$. Tent for assistance with variance analyses of absolu te touching times.

3. A: 45,85 , and $125 \mathrm{deg}$, between two 20-mm-long, 1-mm-diam wires glued to block top; C: 2 -mm-thick circles with 10, 18-, and 24-mm diam; D: 18-mm-diam hole, 3,13 , and $19 \mathrm{~mm}$ deep; H: 10-mm-diam peg, 7, 19, and $31 \mathrm{~mm}$ high; $L$ : length of distance $(7,19$, and $31 \mathrm{~mm})$ between two 7-mm-high, 3-mm-diam pegs; $R$ : Brand 3M sandpaper, Nos. 220,100, and 40 (all smoothed by conductive paint; covered entire block top).

4. Rydberg, S., \& Arnberg, P. W. Overlearning, extinction, and superstition effects on observing behavior in concept learning Psychonomic Science, submitted for publication. 